\title{
Vascularization of Nanohydroxyapatite/Collagen/Poly(L-lactic acid) Composites by Implanting Intramuscularly In Vivo
}

\author{
Hai Wang, ${ }^{1}$ Xiao Chang, ${ }^{1}$ Guixing Qiu, ${ }^{1}$ Fuzhai Cui, ${ }^{2}$ Xisheng Weng, ${ }^{1}$ Baozhong Zhang, \\ Xiaojie Lian, ${ }^{2}$ and Zhihong $\mathrm{Wu}^{1}$ \\ ${ }^{1}$ Department of Orthopaedics, Peking Union Medical College Hospital, No. 1 Shuaifuyuan Hutong, Beijing 100730, China \\ ${ }^{2}$ Biomaterials Laboratory, Department of Material Science \& Engineering, Tsinghua University, Beijing 100084, China
}

Correspondence should be addressed to Zhihong Wu; wuzh3000@126.com

Received 23 January 2014; Accepted 10 February 2014; Published 10 March 2014

Academic Editor: Xiaoming Li

Copyright (C) 2014 Hai Wang et al. This is an open access article distributed under the Creative Commons Attribution License, which permits unrestricted use, distribution, and reproduction in any medium, provided the original work is properly cited.

It still remains a major challenge to repair large bone defects in the orthopaedic surgery. In previous studies, a nanohydroxyapatite/collagen/poly(L-lactic acid) (nHAC/PLA) composite, similar to natural bone in both composition and structure, has been prepared. It could repair small sized bone defects, but they were restricted to repair a large defect due to the lack of oxygen and nutrition supply for cell survival without vascularization. The aim of the present study was to investigate whether nHAC/PLA composites could be vascularized in vivo. Composites were implanted intramuscularly in the groins of rabbits for 2, 6, or 10 weeks $(n=5 \times 3)$. After removing, the macroscopic results showed that there were lots of rich blood supply tissues embracing the composites, and the volumes of tissue were increasing as time goes on. In microscopic views, blood vessels and vascular sprouts could be observed, and microvessel density (MVD) of the composites trended to increase over time. It suggested that nHAC/PLA composites could be well vascularized by implanting in vivo. In the future, it would be possible to generate vascular pedicle bone substitutes with nHAC/PLA composites for grafting.

\section{Introduction}

In the past, autograft, allograft, xenograft, and synthetic bone graft substitute materials have been available for repairing bone defects [1]. Although autologous bone grafting is still the gold standard for osteogenic bone replacement, its inherent shortcomings, including limited availability of bone for harvest and significant donor-site morbidity, restrict its application [2]. Allogenic and xenogenic bone grafts are alternatives, but they are used with reservation because of the risk of disease transmission [3]. Synthetic bone substitute has become technically feasible to repair small sized bone defects in clinical practice, but it is difficult to repair large one [4], because blood supply is restricted in its exterior portion, and cells are the lack of reliable oxygen and nutrient supply. Vascularization plays a very important role in bone repair [5]. It is crucial for tissue-engineered bone (TEB) to establish a vascular network that temporally precedes the formation of new bone [6]. A new bone scaffold material, a nanohydroxyapatite/collagen/poly(L-lactic acid)
(nHAC/PLA) composite, had been developed by biomimetic synthesis before [7-9]. It mimiced the microstructure of cancellous bone. The purpose of this study was to evaluate whether nHAC/PLA composites could be vascularized in vivo.

\section{Materials and Methods}

nHAC/PLA composites were synthesised according to the previous method described before [7-10]. The solution of Type I collagen $(0.67 \mathrm{~g} / \mathrm{L})$ (CELLON Company, Strassen, Luxembourg) was diluted with deionized (DI) water. Solutions of $\mathrm{CaCl}_{2}$ and $\mathrm{H}_{3} \mathrm{PO}_{4}$ were then separately added by drops according to the ratio of $\mathrm{Ca}$ and $\mathrm{P}(\mathrm{Ca} / \mathrm{P}=1.66)$. The solution was adjusted with sodium hydroxide solution to $\mathrm{pH}$ 7.4 at room temperature. After 48 hours, it was centrifugated and freeze-dried. Then, the nHAC deposition was harvested. The nHAC powder was distributed in the PLA matrix $\left(\mathrm{MW}=1.0 \times 10^{5} \mathrm{Da}\right.$; Shandong Medical Appliance Factory, 


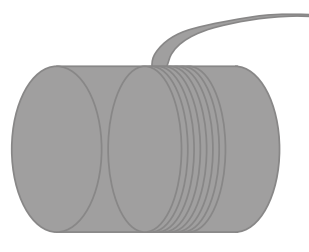

(a)

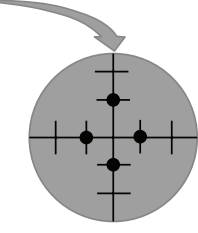

(b)
Figure 1: Schematic drawing demonstrating the approach to histological examinations: (a) 8 cross sections were obtained perpendicular to the long axis in the middle; (b) 4 microphotographs of interest in the inner $1 / 3$ radius at $3,6,9$, and 12 oclock at 400x magnification were evaluated for MVD (dark black points).

Liaocheng, China) at a $1: 1$ weight ratio (nHAC/PLA). The mixture was frozen at $-20^{\circ} \mathrm{C}$ overnight and lyophilized for removing dioxane. After ultrasonication, a cylindrical material was fabricated. The diameter was $10 \mathrm{~mm}$ and the height was $10 \mathrm{~mm}$.

The study was approved by the animal ethic committee of Peking Union Medical College Hospital (PUMCH). All the procedures were conducted in accordance with the guidelines for the care and maintenance of animals. Fifteen three-month-old New Zealand rabbits (Experimental Animal Center of PUMCH, Beijing, China) weighing 2.5 to $3.0 \mathrm{~kg}$ were used. All operations were performed under sterile conditions by the same surgeon. An nHAC/PLA composite was directly implanted into the intramuscular gap in the groin. Histological examinations were performed at 2, 6, and 10 weeks after implantation.

$3 \%$ pentobarbital sodium $(1 \mathrm{~mL} / \mathrm{kg}$ body weight, Sigma, USA) was used for the anesthesia of the animals. 800,000 IU penicillin sodium (North China Pharmaceutical Group Corporation, China) was prophylactically injected before the operation. All the animals were treated as follows. A $2 \mathrm{~cm}$ skin incision from the groin midpoint to the knee was taken, and the myolemma was longitudinally split. A composite was directly placed into the intramuscular gap. The femoral muscle and skin were sutured with 3-0 silk sutures. Postoperatively, 800,000 IU penicillin sodium and $0.15 \mathrm{mg}$ buprenorphine (Tianjing Institute of Pharmaceutical Research, China) were separately administered intramuscularly every 12 hours for 3 days.

The animals were, respectively, sacrificed after 2,6 , and 10 weeks $(n=5 \times 3)$. The composites were removed and fixed in $10 \%$ buffered formalin for 24 hours. After washing, they were decalcified by $14 \%$ ethylenediaminetetraacetic acid (EDTA) solution for 6 weeks. Then, they were dehydrated in graded ethanol and embedded in paraffin. Eight cross sections $(5 \mu \mathrm{m})$ were obtained from each specimen, perpendicular to the long axis in the middle (Figure 1(a)), using a leica microtome (Leica Microsystems, Wetzlar, Germany). For histomorphometric analysis, four sections were randomly selected for haematoxylin and eosin ( $\mathrm{H} \& \mathrm{E})$ staining and microphotographs were taken using a microscope and a digital camera (Leica Microsystems). On each section, four microphotographs of interest in the inner $1 / 3$ radius at 3,6 ,

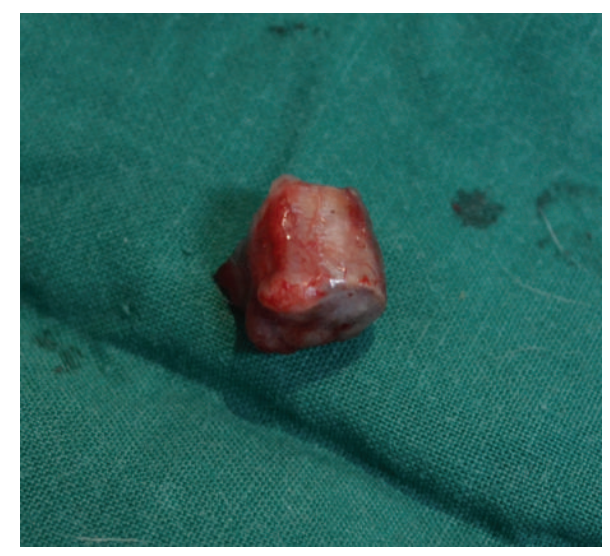

FIGURE 2: Macroscopic views of nHAC/PLA composites.

9, and 12 o'clock at 400x magnification were evaluated, and the number of vessels in high power field (HPF) was counted by two independent and blinded pathologists (Figure 1(b)). Microvessel density (MVD) was calculated for each sample. The results were given as means \pm standard deviation $(\mathrm{x} \pm$ $\mathrm{SD}$, vessels/HPF). Sections of each implant were evaluated for endothelial cell structures by immunofluorescence staining against CD31. The primary antibody was rabbit anti-PECAM1 (Beijing Biosynthesis Biotechnology Corporation, China), and the second antibody was goat anti-rabbit IgG/FITC (Beijing Biosynthesis Biotechnology Corporation, China).

\section{Results}

All rabbits tolerated the surgical procedure and survived well. No perioperative complication, including infection, hematomas, and wound dehiscence, was found. After removing, the implants were surrounded by rich blood supply tissue (Figure 2). As time goes on, the volume of tissue adhering to the implant was also increasing.

Figure 3 showed that there were a lot of inflammatory cells, fibroblasts, blood vessels, and vascular sprouts in the middle of the composites. Along with the time extension, there were fewer inflammatory cells and more fibroblasts and vessels. MVD of the implants was significantly increased (Figure 4, 2 weeks: $6.23 \pm 1.55$ vessels/HPF, 6 weeks: $10.58 \pm$ 2.60 vessels/HPF, and 10 weeks: $12.96 \pm 2.60$ vessels/HPF). The vascular walls of new vessels and red blood cells in the composites were both emphasized as light green fluorescence by CD31 immunofluorescence histochemical staining (Figure 5).

\section{Discussion}

As early as 1763, the importance of blood vessels in bone formation was noted: "the origin of bone is the artery carrying the blood and in it the mineral elements" [11]. Blood vessels are key contributors to the process of osteogenesis, both in development and during repair. Since oxygen and nutrition supply by diffusion are restricted to a maximum range of $200 \mu \mathrm{m}$ into a given matrix [12] and suboptimal initial 


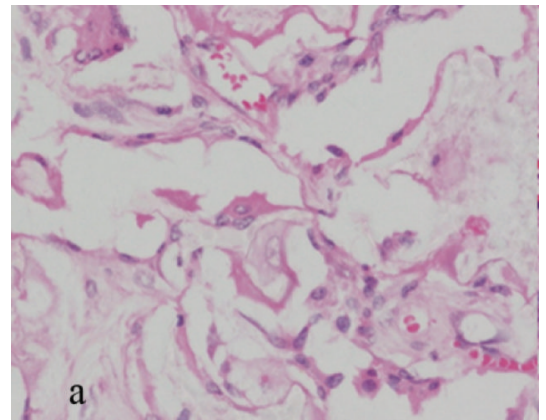

(a)

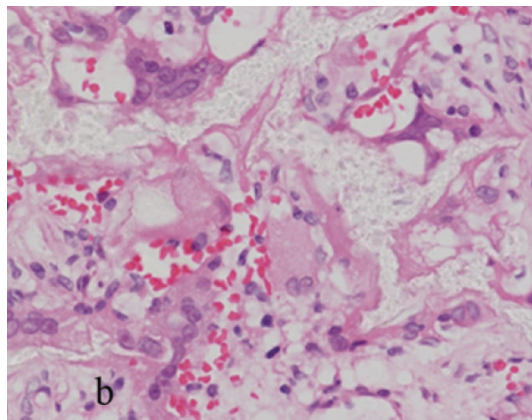

(b)

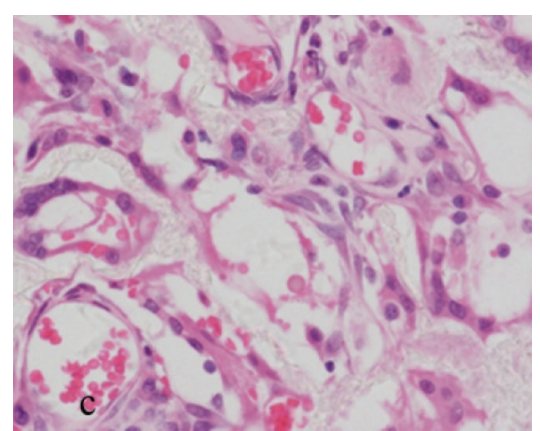

(c)

FIGURE 3: Hematoxylin and eosin (H \& E) staining of specimens (magnification $\times 400$ ): (a) 2 weeks, (b) 6 weeks, and (c) 10 weeks.

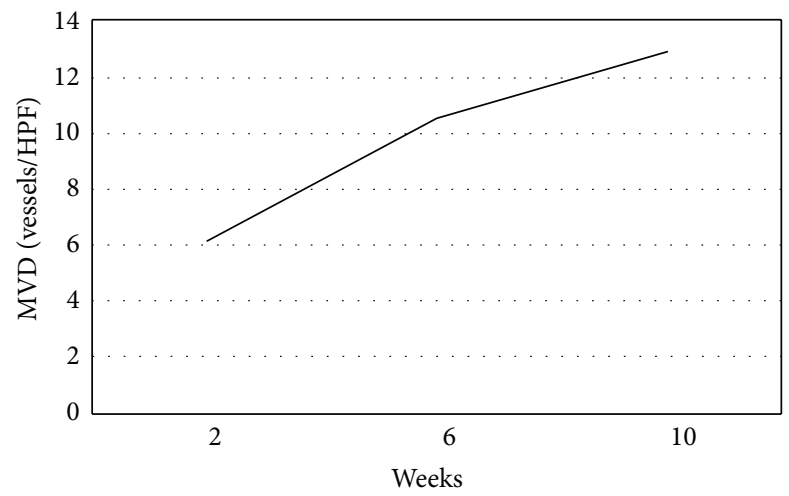

Figure 4: Microvessel density (MVD) of the composites.

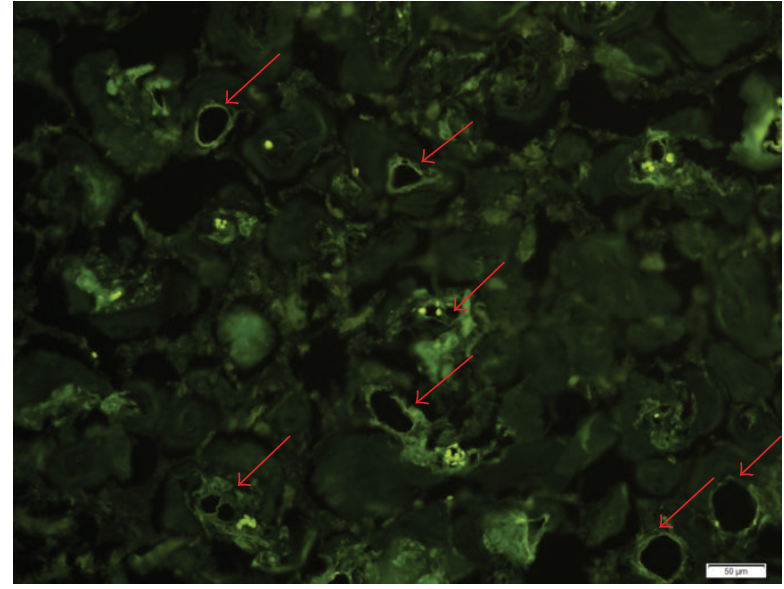

FIGURE 5: CD31 immunofluorescence histochemical staining of specimens by 10 weeks after implantation (magnification $\times 200$ ): the vascular walls and red blood cells display remarkable light green fluorescence.

vascularization often limits survival of cells in the center of large constructs [13], therefore, the vascularization of TEB has drawn scholars' interests recently. It includes the progresses of angiogenesis (the sprouting of capillaries from preexisting blood vessels) and vasculogenesis (the assembly of capillaries in situ from undifferentiated endothelial cells)
[14]. Angiogenesis is involved in the initiation and promotion of endochondral and intramembranous ossification in bone growth and remodeling [15].

Nowadays, the technology of constructing a blood vessel system has still many difficult issues that cannot be resolved by the development of tissue engineering alone. Surgical angiogenesis is the unique promising method to establish a functional vascular network. It utilizes the preexisting blood vessels as a vascular carrier to regenerate nutrient vessels by incorporating artificial materials and cells into them.

The most frequent method of neovascularization in tissue engineering is that the neovascular bed originates from the periphery of the construct implanted into a site of high vascularization potential (subcutaneous [16], intramuscular [17], and intraperitoneal [13]). Vascularization occurs via an endogenous response to the surgical implantation, which creates an inflammatory wound-healing response [14]. Endogenous angiogenic growth factors are expressed because of the hypoxia of the implant. Exogenous angiogenic growth factors can be incorporated into the implant to enhance the vascularization. Warnke et al. reported that they had successfully used intramuscularly as in vivo bioreactor for prefabrication of a large mandible replacement in a clinical practice [18].

Tissue engineering is promising therapeutic strategies for the repairment of diseased or injured tissues and organs [19]. There are three elements in tissue engineering, including 
scaffolds, seeding cells, and cytokines. Scaffolds play an essential role, because they not only supported and regulated the growth of cells, but also guided the ingrowth of periphery tissue [20-22]. The osteogenesis and angiogenesis abilities of scaffolds have been significantly developed [21]. The advantage of biocompatibility and osteoinduction of microstructured calcium phosphate materials has been shown before [23, 24]. Similar to the natural cancellous bone in main composition and in hierarchical microstructure, a new bionic porous scaffold, nHAC/PLA composite, has been fabricated before [9]. Its pore size was about $100-300 \mu \mathrm{m}$, and its porosity was about $80 \%$. Cellular activities were affected by chemical composition, conformation, porosity, and hydrophobicity of matrix [25]. The osteogenesis ability of nHAC/PLA composites were confirmed by osteoblasts culture and animal model tests [9]. But its vascularization ability did not have been estimated before. This might decide its availability for repairing large bone defects. In this study, we first demonstrated that it could guide vessels' ingrowth in vivo to achieve its vascularization.

In conclusion, the ability of nHAC/PLA composites was shown to generate new vascular networks for prefabrication of large bone substitutes by implanting intramuscularly. A vascularized large bone flap for transplanting could be prefabricated using a nHAC/PLA composite as a scaffold in the future.

\section{Conflict of Interests}

The authors have declared that there is no conflict of interests.

\section{Authors' Contribution}

Hai Wang and Xiao Chang are cofirst authors.

\section{Acknowledgment}

This work was supported by National Natural Science Foundation of China (NSFC no. 81000802).

\section{References}

[1] T. W. Bauer and G. F. Muschler, "Bone graft materials: an overview of the basic science," Clinical Orthopaedics and Related Research, no. 371, pp. 10-27, 2000.

[2] J. P. Beier, R. E. Horch, A. Hess et al., "Axial vascularization of a large volume calcium phosphate ceramic bone substitute in the sheep AV loop model," Journal of Tissue Engineering and Regenerative Medicine, vol. 4, no. 3, pp. 216-223, 2010.

[3] A. J. Aho, T. Ekfors, P. B. Dean, H. T. Aro, A. Ahonen, and V. Nikkanen, "Incorporation and clinical results of large allografts of the extremities and pelvis," Clinical Orthopaedics and Related Research, no. 307, pp. 200-213, 1994.

[4] C. A. Vacanti, L. J. Bonassar, M. P. Vacanti, and J. Shufflebarger, "Replacement of an avulsed phalanx with tissue-engineered bone," The New England Journal of Medicine, vol. 344, no. 20, pp. 1511-1514, 2001.

[5] L.-L. Ren, D.-Y. Ma, X. Feng, T.-Q. Mao, Y.-P. Liu, and Y. Ding, "A novel strategy for prefabrication of large and axially vascularized tissue engineered bone by using an arteriovenous loop," Medical Hypotheses, vol. 71, no. 5, pp. 737-740, 2008.

[6] H. Yu, P. J. VandeVord, L. Mao, H. W. Matthew, P. H. Wooley, and S.-Y. Yang, "Improved tissue-engineered bone regeneration by endothelial cell mediated vascularization," Biomaterials, vol. 30, no. 4, pp. 508-517, 2009.

[7] C. Du, F. Z. Cui, W. Zhang, Q. L. Feng, X. D. Zhu, and K. de Groot, "Formation of calcium phosphate/collagen composites through mineralization of collagen matrix," Journal of Biomedical Materials Research, vol. 50, no. 4, pp. 518-527, 2000.

[8] C. Du, F. Z. Cui, X. D. Zhu, and K. de Groot, "Threedimensional nano-HAp/collagen matrix loading with osteogenic cells in organ culture," Journal of Biomedical Materials Research, vol. 44, no. 4, pp. 407-415, 1999.

[9] S. S. Liao, F. Z. Cui, W. Zhang, and Q. L. Feng, "Hierarchically biomimetic bone scaffold materials: nano-HA/collagen/PLA composite," Journal of Biomedical Materials Research Part B, vol. 69, no. 2, pp. 158-165, 2004.

[10] J. Li, J. Hong, Q. Zheng et al., "Repair of rat cranial bone defects with nHAC/PLLA and BMP-2-related peptide or rhBMP-2," Journal of Orthopaedic Research, vol. 29, no. 11, pp. 1745-1752, 2011.

[11] R. A. D. Carano and E. H. Filvaroff, "Angiogenesis and bone repair," Drug Discovery Today, vol. 8, no. 21, pp. 980-989, 2003.

[12] A. S. Goldstein, T. M. Juarez, C. D. Helmke, M. C. Gustin, and A. G. Mikos, "Effect of convection on osteoblastic cell growth and function in biodegradable polymer foam scaffolds," Biomaterials, vol. 22, no. 11, pp. 1279-1288, 2001.

[13] U. Kneser, P. M. Kaufmann, H. C. Fiegel et al., "Long-term differentiated function of heterotopically transplanted hepatocytes on three-dimensional polymer matrices," Journal of Biomedical Materials Research, vol. 47, no. 4, pp. 494-503, 1999.

[14] O. C. S. Cassell, S. O. P. Hofer, W. A. Morrison, and K. R. Knight, "Vascularization of tissue-engineered grafts: the regulation of angiogenesis in reconstructive surgery and in disease states," British Journal of Plastic Surgery, vol. 55, no. 8, pp. 603-610, 2002.

[15] J. M. Kanczler and R. O. C. Oreffo, "Osteogenesis and angiogenesis: the potential for engineering bone," European Cells and Materials, vol. 15, pp. 100-114, 2008.

[16] U. Kneser, A. Voogd, J. Ohnolz et al., "Fibrin gel-immobilized primary osteoblasts in calcium phosphate bone cement: in vivo evaluation with regard to application as injectable biological bone substitute," Cells Tissues Organs, vol. 179, no. 4, pp. 158169, 2005.

[17] J. P. Beier, U. Kneser, J. Stern-Sträter, G. B. Stark, and A. D. Bach, "Y chromosome detection of three-dimensional tissueengineered skeletal muscle constructs in a syngeneic rat animal model," Cell Transplantation, vol. 13, no. 1, pp. 45-53, 2004.

[18] P. Warnke, I. Springer, P. J. Wiltfang et al., "Growth and transplantation of a custom vascularised bone graft in a man," The Lancet, vol. 364, no. 9436, pp. 766-770, 2004.

[19] X. Li, Y. Yang, Y. Fan, Q. Feng, F. Z. Cui, and F. Watari, "Biocomposites reinforced by fibers or tubes as scaffolds for tissue engineering or regenerative medicine," Journal of Biomedical Materials Research Part A, 2013.

[20] R. Murugan and S. Ramakrishna, "Nano-featured scaffolds for tissue engineering: a review of spinning methodologies," Tissue Engineering, vol. 12, no. 3, pp. 435-447, 2006.

[21] X. Li, H. Gao, M. Uo et al., "Effect of carbon nanotubes on cellular functions in vitro," Journal of Biomedical Materials Research Part A, vol. 91, no. 1, pp. 132-139, 2009. 
[22] X. Li, H. Liu, X. Niu et al., "The use of carbon nanotubes to induce osteogenic differentiation of human adipose-derived MSCs in vitro and ectopic bone formation in vivo," Biomaterials, vol. 33, no. 19, pp. 4818-4827, 2012.

[23] X. Li, L. Wang, Y. Fan, Q. Feng, F. Z. Cui, and F. Watari, "Nanostructured scaffolds for bone tissue engineering," Journal of Biomedical Materials Research Part A, vol. 101, no. 8, pp. 24242435, 2013.

[24] G. Zhou, Y. Hou, L. Liu et al., "Preparation and characterization of NiW-nHA composite catalyst for hydrocracking," Nanoscale, vol. 4, no. 24, pp. 7698-7703, 2012.

[25] G. Zhou, G. Zhang, Z. Wu et al., "Research on the structure of fish collagen nanofibers influenced cell growth," Journal of Nanomaterials, vol. 2013, Article ID 764239, 6 pages, 2013. 

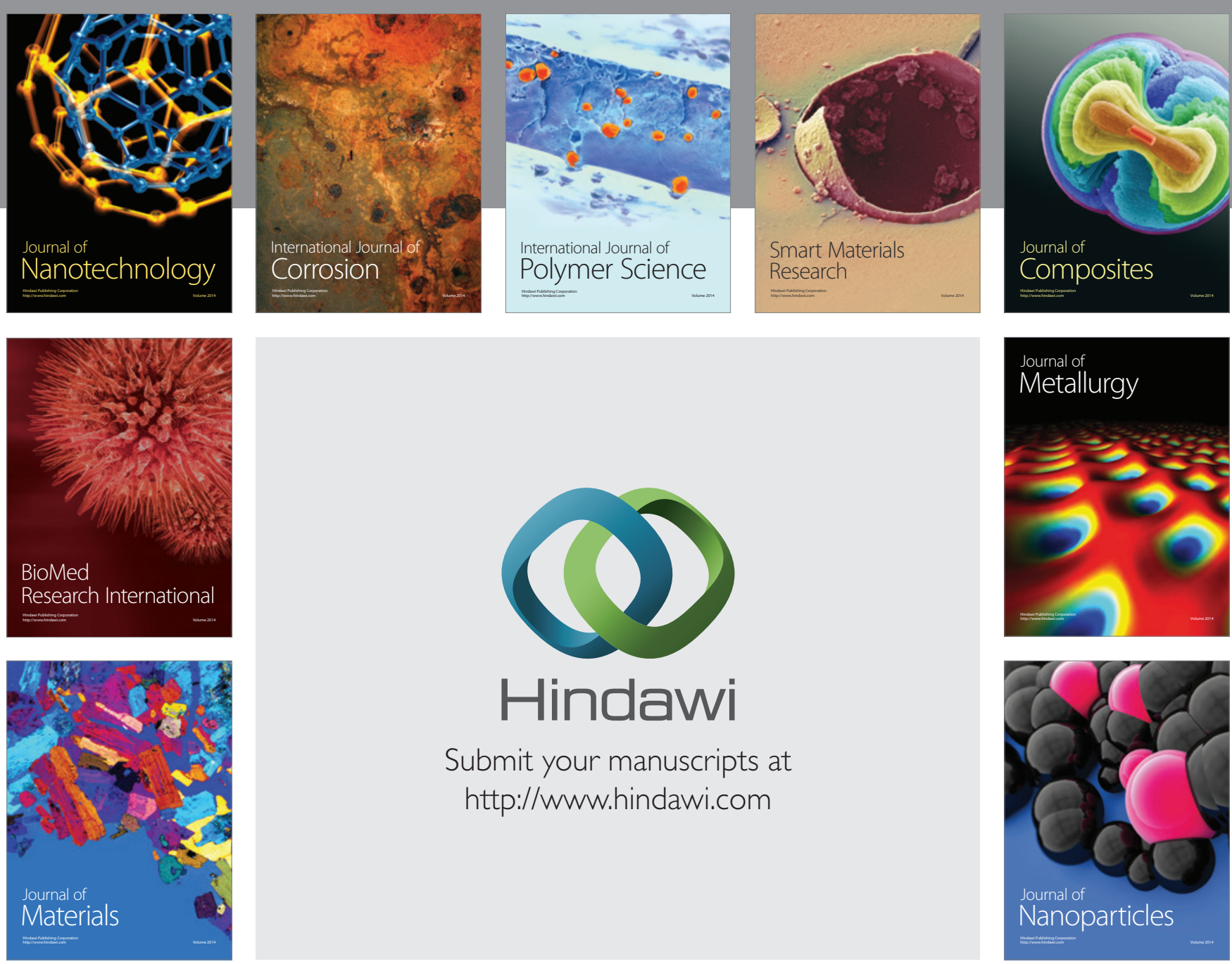

Submit your manuscripts at http://www.hindawi.com
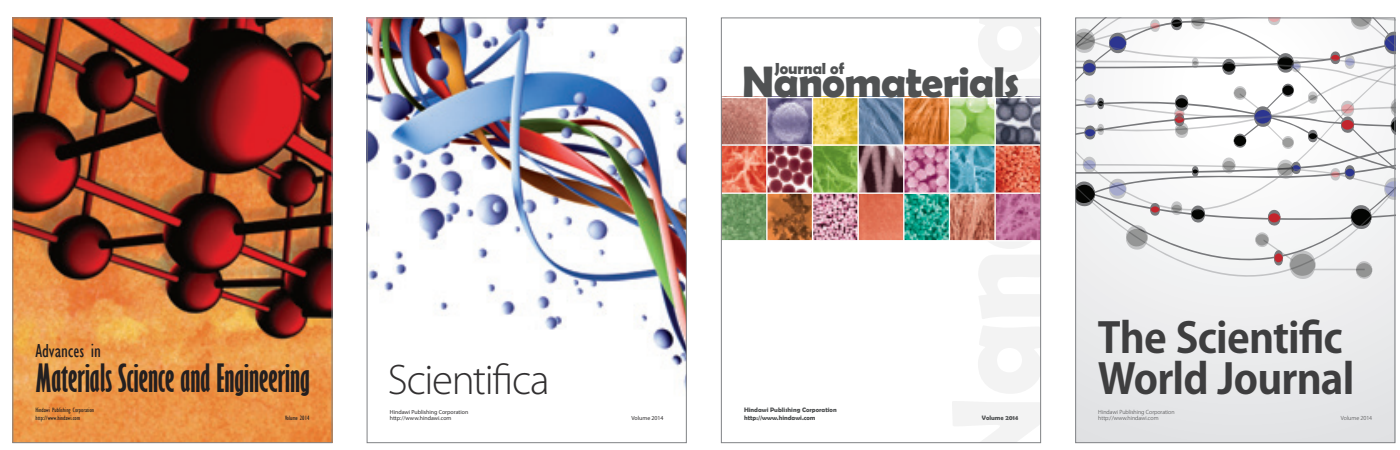

\section{The Scientific World Journal}
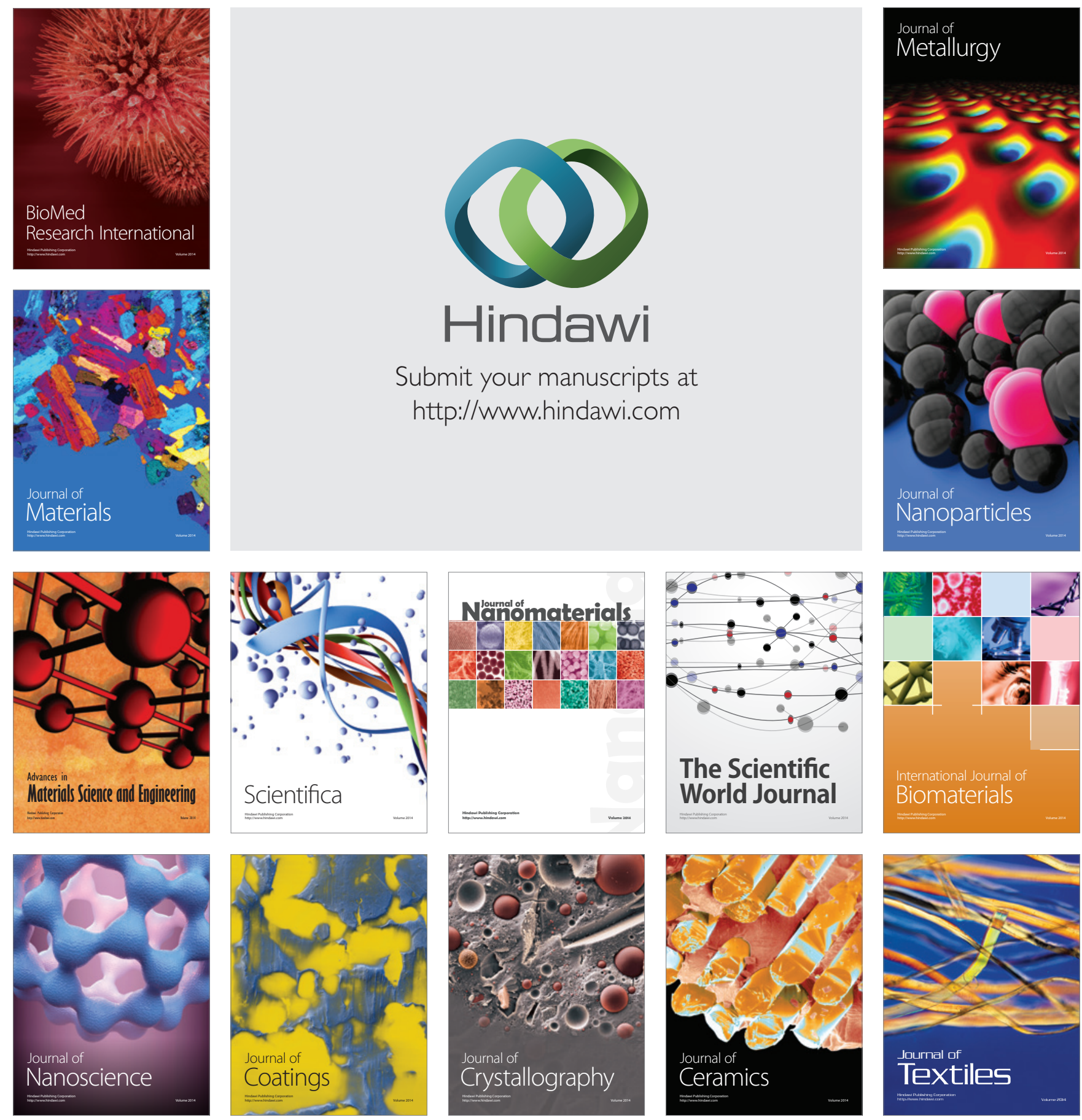